Methods We conducted a cross-sectional study of supervisors and workers from 30 randomly selected companies in Manitoba and Northwestern Ontario, Canada. Participating companies required a minimum of 50 employees. We distributed two separate surveys to each company: one to supervisors and one to workers. We asked all supervisors to complete a webbased survey including a case vignette of a worker with a mental health disorder and a number of scales assessing factors that may affect their decisions to provide workplace accommodations. We also asked all workers to complete a web-based survey indicating whether or not they suffer from a MHD and whether accommodations were offered. We also assessed individual perspectives and group-level information on the workplace, supervisor, and worker factors through additional scales on the surveys.

Results Data collection is expected to be completed in the fall, 2017. We have currently collected data from fourteen of the thirty companies including 130 supervisors and 330 workers. In our presentation, we will identify factors associated with supervisors' decisions to accommodate workers with MHD.

Conclusion The findings will be important for all work disability prevention stakeholders as they will identify important targets for intervention to improve accommodations for workers with MHD.

\section{A SUPERVISOR TRAINING PROGRAM FOR WORK DISABILITY PREVENTION: PRELIMINARY RESULTS FROM A CLUSTER RANDOMISED CONTROLLED TRIAL}

\begin{abstract}
1,2,3,4 VL Kristman* ${ }^{1} \mathrm{JJ}$ Armstrong, ${ }^{5}$ WS Shaw. ${ }^{1}$ Department of Health Sciences, Lakehead University, Thunder Bay, Ontario, Canada; ${ }^{2}$ Division of Human Sciences, Northern Ontario School of Medicine, Thunder Bay, Ontario, Canada; ${ }^{3}$ Institute for Work and Health, Toronto, Ontario, Canada; ${ }^{4}$ Dalla Lana School of Public Health, University of Toronto, Toronto, Ontario, Canada; ${ }^{5}$ Department of Family Medicine and Community Health, University of Massachusetts Medical School, Worcester, Massachusetts, USA
\end{abstract}

\subsection{6/oemed-2018-ICOHabstracts. 1587}

Introduction Providing supervisors with tools to improve their response to workplace injuries or illnesses may improve disability outcomes. The objective of this study was to examine the effectiveness of the Supervisor/Manager Accommodation Recognition and Training (SMART) Program on reducing the total duration of workers' lost-time claims. Here, we provide preliminary results from two Canadian (located in Ontario and British Columbia) and one American employer.

Methods Within each organisation, work units were randomly selected to have their supervisors receive the training program. Work units not assigned to the program served as the control group for the study. Work disability outcome data were oneyear prior to and one-year post training for comparison purposes. Web based surveys were used to collect information on supervisors' knowledge and responses to workplace injuries at baseline, 3- and 6 months post training.

Results For the Ontario-based employer, the SMART program did not impact the total duration of workers' lost-time claims when compared to the controls. For the British Columbiabased employer, trained work sites had a reduction in both the number of days off per injury incident $(-6.2)$ and the number of short-term disability claims per 100 workers $(-10.5)$. Across the American-based employer, the work sites that received the SMART training had a significant reduction in the number of days off per workers' compensation claim $(-4.9)$, a small decrease in the average number of days per short-term disability claim $(-2.7)$ and the number of Workers' Compensation claims were reduced to half the rate post-training (8.3 claims per 100 employees per year down to 4.4). Survey results will also be discussed.

Conclusion The mixed results of the preliminary data highlight the importance of context when studying complex organisations. Employee culture, policies and practices of management, type of industry, and other organisational factors have a strong influence on work disability outcomes.

\section{THE RELATIONSHIP BETWEEN EARLY PRESCRIPTION DISPENSING PATTERNS AND WORK DISABILITY IN A COHORT OF LOW BACK PAIN WORKERS' COMPENSATION CLAIMANTS}

\begin{abstract}
${ }^{1}$ Nancy Carnide, ${ }^{1,2,3}$ Sheilah Hogg-Johnson, ${ }^{1,4}$ Mieke Koehoorn, ${ }^{1,5,6}$ Andrea Furlan, 2,3,7Pierre Côté. 'Institute for Work and Health, Toronto, Canada; ${ }^{2}$ Canadian Memorial Chiropractic College, Toronto Canada; ${ }^{3}$ Dalla Lana School of Public Health, University of Toronto, Toronto, Canada; ${ }^{4}$ School of Population and Public Health, Faculty of Medicine, University of British Columbia, Vancouver, Canada; ${ }^{5}$ Toronto Rehabilitation Institute, University Health Network, Toronto, Canada; ${ }^{6}$ Department of Medicine, Faculty of Medicine, University of Toronto, Toronto, Canada; ${ }^{7}$ Faculty of Health Sciences, University of Ontario Institute Of Technology, Oshawa, Canada
\end{abstract}

10.1136/oemed-2018-ICOHabstracts. 1588

Introduction Opioids are prescribed early among workers' compensation claimants with low back pain (LBP), despite little evidence supporting this practice. The comparative effect of early opioids relative to other medications on work disability is unknown. The objective was to examine the association between prescription opioids, nonsteroidal anti-inflammatory drugs (NSAIDs), and skeletal muscle relaxants (SMRs) dispensed in the first eight weeks following a compensated, work-related low back injury and work disability.

Methods Historical cohort study of workers' compensation claimants in British Columbia, Canada filing a short-term claim for a LBP injury. Exposure variables were constructed using dispensing data from the first eight weeks after injury: drug class(es) dispensed, drug class(es) at first dispense, days supply, strength of opioids dispensed, and average daily morphine-equivalent dose (MED). Two outcomes (time on benefits, any benefit receipt) after eight weeks and up to one year were considered.

Results Compared to claimants receiving NSAIDs and/or SMRs only, the incidence rate ratio (IRR) of days on benefits for claimants dispensed opioids only or opioids with NSAIDs and/ or SMRs was 1.09 (95\% CI: 1.04 to 1.14) and $1.26(95 \%$ CI: 1.22 to 1.30$)$, respectively. The IRR for claimants dispensed strong opioids only or strong and weak opioids combined was 1.21 (95\% CI: 1.12 to 1.30 ) and 1.29 (95\% CI: 1.20 to 1.39), respectively, compared to weak opioids only. Each 7 day increase in supply of opioids, NSAIDs, and SMRs resulted in a $10 \%, 4 \%$, and $3 \%$ increase, respectively, in rate of days on benefits. Each $30 \mathrm{mg} /$ day increase in daily MED resulted in a $4 \%$ increase in rate of days on benefits. Larger effect sizes were seen for benefit receipt outcome.

Discussion Findings suggest early opioid provision has an adverse effect on work disability. However, residual confounding likely accounts for some of the findings. Research is needed that accounts for prescriber, system, and workplace factors and elucidates the mechanism underlying the relationship. 